\title{
Toroidal Seifert fibered surgeries on Montesinos knots
}

\author{
KAZUhiro ICHIHARA AND In DAE Jong
}

\begin{abstract}
We show that if a Montesinos knot admits a Dehn surgery yielding a toroidal Seifert fibered 3-manifold, then the knot is the trefoil knot and the surgery slope is 0 .
\end{abstract}

\section{Introduction}

Let $K$ be a knot in the 3 -sphere $S^{3}$ and $E(K)$ the exterior of $K$. For a slope $\gamma$ on the boundary of $E(K)$, we denote by $K(\gamma)$ the manifold obtained by the Dehn surgery on $K$ along a slope $\gamma$, i.e., $K(\gamma)$ is obtained by gluing a solid torus $V$ to $E(K)$ so that a simple closed curve representing $\gamma$ bounds a disk in $V$. We call such a slope $\gamma$ the surgery slope. It is well known that a slope on the boundary torus $\partial E(K)$ is parameterized by an element of $\mathbb{Q} \cup\{1 / 0\}$ by using the standard meridian-longitude system for $K$. Thus, when a slope $\gamma$ corresponds to $r \in \mathbb{Q} \cup\{1 / 0\}$, we call the Dehn surgery along $\gamma$ the $r$-surgery for brevity, and denote $K(\gamma)$ by $K(r)$. Since $K(1 / 0)$ is homeomorphic to $S^{3}$ again, $1 / 0$-surgery is called the trivial surgery. See $[17,28]$ for basic references.

The hyperbolic Dehn surgery theorem, established by Thurston [29, Theorem 5.8.2], says that each hyperbolic knot admits only finitely many Dehn surgeries yielding non-hyperbolic manifolds. Here a knot is called hyperbolic if its complement admits a complete hyperbolic structure of finite volume. Thereby, a Dehn surgery on a hyperbolic knot is called exceptional if it yields a non-hyperbolic manifold. As a consequence of the geometrization conjecture, raised by Thurston [30, Section 6 , question 1], and established by Perelman's works [25-27], exceptional surgeries are classified into the three types: a Seifert fibered surgery a toroidal surgery and a reducible surgery. Here a Dehn surgery is called Seifert fibered/toroidal/reducible if it yields a Seifert fibered/toroidal/reducible manifold. We refer the reader to [3] for a survey.

We here note that the classification is not exclusive, that is, there exist Seifert fibered 3-manifolds which are both toroidal and reducible. However, 


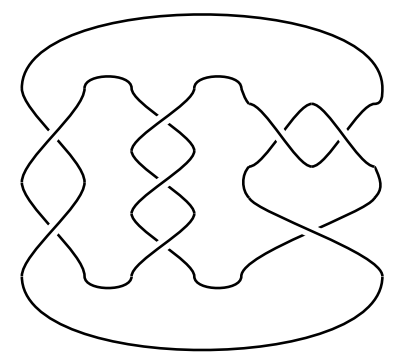

Figure 1: $M(1 / 2,1 / 3,-2 / 3)$.

hyperbolic knots in $S^{3}$ are conjectured to admit no reducible surgeries. This is the well known, but still open, Cabling Conjecture [9]. Thus, we consider in this paper a Dehn surgery on a knot in $S^{3}$ yielding a 3-manifold which is toroidal and Seifert fibered, which we call a toroidal Seifert fibered surgery.

Actually there exist infinitely many hyperbolic knots in $S^{3}$ each of which admits a toroidal Seifert fibered surgery. These were found by EudaveMuñoz [6, Proposition 4.5(1) and (3)], and Gordon and Luecke [10] independently. On the other hand, Motegi [21] studied toroidal Seifert fibered surgeries on symmetric knots, and gave several restrictions on the existence of such surgeries. In particular, he showed that only the trefoil knot admits a toroidal Seifert fibered surgery among two-bridge knots [21, Corollary 1.6].

Extending this result, in this paper, we show the following:

Theorem 1.1. Let $K$ be a Montesinos knot in the 3-sphere. If $K$ admits a toroidal Seifert fibered surgery, then $K$ is the trefoil knot and the surgery slope is 0 .

A Montesinos knot of type $\left(R_{1}, \ldots, R_{l}\right)$, denoted by $M\left(R_{1}, \ldots, R_{l}\right)$, is defined as a knot admitting a diagram obtained by putting rational tangles $R_{1}, \ldots, R_{l}$ together in a circle (see figure 1 ). For brevity, here and in the sequel, we abuse $R_{i}$ to denote a rational number (or, an irreducible fraction) or the corresponding rational tangle depending on the context. The minimal number of such rational tangles is called the length of a Montesinos knot. In particular, a Montesinos knot $K$ is called a pretzel knot of type $\left(a_{1}, \ldots, a_{l}\right)$, denoted by $P\left(a_{1}, \ldots, a_{l}\right)$, if the rational tangles in $K$ are of the form $1 / a_{1}, \ldots, 1 / a_{l}$.

Exceptional surgeries on Montesinos knots are extensively studied. See $[13,32-34]$ for example. 
On toroidal Seifert fibered surgeries, in our forthcoming paper [14], we will also show that among prime alternating knots, only the trefoil knot admits a toroidal Seifert fibered surgery.

\section{Proof of Theorem 1.1}

In order to prove Theorem 1.1, we will show the following two lemmas in Sections 3 and 4 , respectively.

Lemma 2.1. For the pretzel knot $K_{m, n}=P(2 m, 2 n+1,-2 n-1)$ with integers $m \neq 0$ and $n \geq 1$, the surgered manifold $K_{m, n}(0)$ is not Seifert fibered.

Lemma 2.2. For the Montesinos knot $K_{ \pm}=M(-1 / 2,1 / 3,1 /(6 \pm 1 / 2))$, the surgered manifold $K_{ \pm}(0)$ is not Seifert fibered.

Assuming these lemmas, we prove Theorem 1.1.

Proof of Theorem 1.1. Let $K$ be a Montesinos knot admitting a toroidal Seifert fibered surgery. That is, we suppose that $K(r)$ is a toroidal Seifert fibered manifold for some $r \in \mathbb{Q}$. Then the length of $K$ must be less than four since Montesinos knots of length at least four admit no exceptional surgeries [32]. On the other hand, if the length of $K$ is less than three, meaning that $K$ is a two-bridge knot, then $K$ is the trefoil knot and $r=0$, i.e., the surgery slope is longitudinal [21, Corollary 1.6].

Thus, in the following, we suppose that $K$ is of length 3 . In addition, if $K$ is non-hyperbolic, then $K$ is either $P(-2,3,3)$ or $P(-2,3,5)$, which are actually the torus knots of type $(3,4)$ or of type $(3,5)$. This was originally shown by Oertel [24, Corollary 5] together with the result in the unpublished monograph by Bonahon and Siebenmann [1, 2]. Dehn surgeries on torus knots are completely classified [20], and the two torus knots admit no toroidal Seifert fibered surgeries. Thus, from now on, we further assume that $K$ is hyperbolic. Then we have the following:

Claim 2.1. The knot $K$ must be one of the following:

(I) $P(2 m, 2 n+1,-2 n-1)$ for integers $m \neq 0$ and $n \geq 1$.

(II) $M(-1 / 2,1 / 3,1 /(6 \pm 1 / 2))$.

Furthermore the slope $r$ must be 0 . 
Proof. Since the exterior of a Montesinos knot of length three contains no closed essential surface [24, Corollary 4], if $K(r)$ is toroidal, then $r$ must be a boundary slope of an essential surface. Furthermore, if $K(r)$ is Seifert fibered, then by [15, Proposition 1], it follows that $K$ is fibered and $r=0$.

On the other hand, toroidal surgeries on hyperbolic Montesinos knots of length three are completely listed by $\mathrm{Wu}$ [34]. Thus, the candidates for toroidal Seifert fibered surgeries on Montesinos knots are 0-surgeries on fibered knots contained in Wu's list. The 0-surgeries contained in Wu's list are the following:

(i) The 0 -surgery on $P\left(2 p_{1}-1,2 p_{2}-1,2 p_{3}-1\right)$ for integers $p_{i} \neq 0,1$.

(ii) The 0-surgery on $P(2 m, 2 n+1,-2 n-1)$ for integers $m \neq 0$ and $n \geq 1$.

(iii) The 0 -surgery on $M(-1 / 2,1 / 3,1 /(6+1 / 2 s))$ for an integer $s \neq 0$.

Notice that the first class of pretzel knots are of genus one. It is known that a genus one fibered knot is either the trefoil knot or the figure-eight knot [8]. Therefore, $P\left(2 p_{1}-1,2 p_{2}-1,2 p_{3}-1\right)$ for integers $p_{i} \neq 0,1$ is not fibered.

On the third case, calculating the Alexander polynomial, we have

$$
\Delta_{M(-1 / 2,1 / 3,1 /(6+1 / 2 s))}(t)=-s\left(t^{2}+t^{-2}\right)+2 s+1
$$

Since the Alexander polynomials of fibered knots have to be monic (see [28] for example), if the knot $M(-1 / 2,1 / 3,1 /(6+1 / 2 s))$ is fibered, then $s= \pm 1$. This completes the proof of Claim 2.1.

However, the 0-surgeries on the knots listed in Claim 2.1 are not Seifert fibered surgeries by Lemmas 2.1 and 2.2, respectively. Therefore, a Montesinos knot of length three admits no toroidal Seifert fibered surgery, and this completes the proof of Theorem 1.1.

Remark 2.1. By using Gabai's arguments [7], we see that each of the knots listed in Claim 2.1 is fibered.

Let $\mathbf{B}_{l}\left(S^{2}\right)$ be the $l$-string braid group on the 2 -sphere. We denote by $\sigma_{1}, \ldots, \sigma_{l-1}$ the standard generators of $\mathbf{B}_{l}\left(S^{2}\right)$. See [23] for details about braids on the 2-sphere. The following lemma is used in both Sections 3 and 4. 
Lemma 2.3. Let $\widehat{b}$ and $\widehat{b^{\prime}}$ be the closed braids for $b \in \mathbf{B}_{l}\left(S^{2}\right)$ and $b^{\prime} \in$ $\mathbf{B}_{l^{\prime}}\left(S^{2}\right)$, respectively. If $\widehat{b}$ is isotopic to $\widehat{b^{\prime}}$ in $S^{2} \times S^{1}$, then the numbers of strands of $b$ and $b^{\prime}$ coincide, i.e., $l=l^{\prime}$ holds. Furthermore, with respect to the standard generators, the exponent sum of $b$ is congruent to that of $b^{\prime}$ modulo $2(l-1)$.

Proof. It is a well-known fact that $\widehat{b}$ is isotopic to $\widehat{b^{\prime}}$ in $S^{2} \times S^{1}$ if and only if $b$ and $b^{\prime}$ are both $l$-strand braids on the 2 -sphere for some positive integer $l$, and $b$ is conjugate to $b^{\prime}$ in $\mathbf{B}_{l}\left(S^{2}\right)$. See [22, Section 3] for example. Furthermore, by [23, Chapter 11, Proposition 2.3], if $b$ is conjugate to $b^{\prime}$ in $\mathbf{B}_{l}\left(S^{2}\right)$, then the exponent sum of $b$ is congruent to that of $b^{\prime}$ modulo $2(l-1)$.

\section{On pretzel knots}

In this section, we prove the following:

Lemma 3.1. For the pretzel knot $K_{m, n}=P(2 m, 2 n+1,-2 n-1)$ with integers $m \neq 0$ and $n \geq 1$, the surgered manifold $K_{m, n}(0)$ is not Seifert fibered.

Proof. We note that the knot $K_{m, n}=P(2 m, 2 n+1,-2 n-1)$ is the mirror image of the knot $K_{-m, n}=P(-2 m, 2 n+1,-2 n-1)$. Thus, if $K_{m, n}(0)$ is not Seifert fibered for $m \geq 1$, then $K_{-m, n}(0)$ is also not Seifert fibered.

We first consider the case where $n=1$ as follows.

Claim 3.1. The surgered manifold $K_{m, 1}(0)$ is not Seifert fibered.

Proof. Applying the Montesinos trick [19], the surgered manifold $K_{m, 1}(0)$ is homeomorphic to the double-branched covering space of $S^{3}$ branched along the two-component link $\lambda_{m}$ depicted in figure 2. In the decomposition of

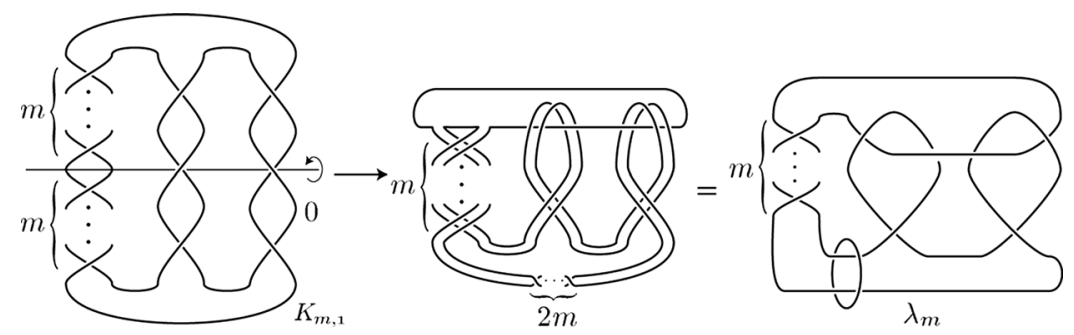

Figure 2: The Montesinos trick for $K_{m, 1}(0)$. 

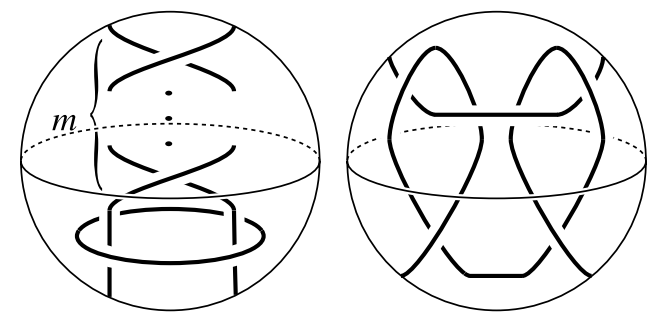

Figure 3: A tangle decomposition of $\lambda_{m}$.

the link $\lambda_{m}$ as a partial sum of two 2-string tangles, the left part can be viewed as a Montesinos tangle or a Montesinos-m tangle in the sense of [6], see figure 3. Correspondingly, the double-branched cover of the left tangle is either a Seifert fibered space over the disk with two exceptional fibers of indices 2 or a Seifert fibered space over the Möbius band without exceptional fibers. In either of these cases, it is verified that their Seifert fibrations do not match since $m \neq 0$ (see [2, Appendix A; 16, Chapter IV], for example). Thus, $K_{m, 1}(0)$ is not Seifert fibered.

Suppose that $K_{m, n}(0)(n \geq 2)$ is Seifert fibered. As illustrated in the upper half of figure 4 , let $\iota$ be a strong inversion of $K_{m, n}$ with respect to an axis $\alpha$, and $F$ a non-orientable spanning surface invariant under $\iota$. Let $V$ be the attached solid torus via the 0 -surgery. Then there exists a meridian

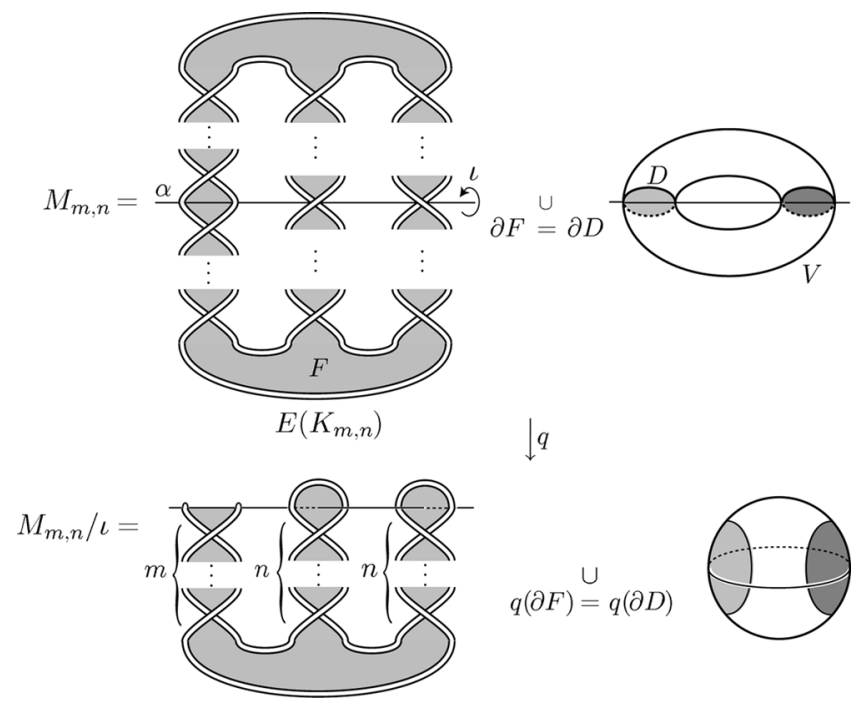

Figure 4: The Montesinos trick for $K_{m, n}(0)$. 
disk $D$ in $V$ such that $F \cup D$ gives a Klein bottle $\widehat{F}$ embedded in $K_{m, n}(0)$. For a manifold $X$ and a sub-manifold $Y$, we denote by $N(Y)$ a regular neighborhood of $Y$ in $X$ and by $N^{\circ}(Y)$ the interior of $N(Y)$.

For considering the remaining cases where $n \geq 2$, the following is the key claim.

Claim 3.2. If $K_{m, n}(0)$ is Seifert fibered, then $K_{m, n}(0)-N^{\circ}(\widehat{F})$ is also Seifert fibered.

Proof. Let $M_{m, n}=K_{m, n}([\partial F])=K_{m, n}(0)$. We can naturally extend the involution $\iota$ on $E\left(K_{m, n}\right)$ to that on $M_{m, n}$, denoted by $\widehat{\iota}$, with the axis $\widehat{\alpha}$ appearing as the natural extension of $\alpha$. We may assume that $\widehat{F}$ is invariant under $\widehat{\iota}$. Then, as shown in [34], the torus appearing as $\partial N(\widehat{F})$ is incompressible in $M_{m, n}$. By [16, VI.34. Theorem], such a torus is isotoped so that it is saturated in a Seifert fibration of $M_{m, n}$. That is, the 3-manifold $M_{m, n}-N^{\circ}(\widehat{F})$ admits a Seifert fibration.

Then $M_{m, n} / \widehat{\iota}=S^{3}$ is described as shown in the lower half of figure 4 . Let $q: M_{m, n} \rightarrow S^{3}$ be the quotient map with respect to the involution $\widehat{\iota}$. Removing $N^{\circ}(q(\widehat{F}))$, that is, an open regular neighborhood of the pale shaded disk in the lower half of figure 4 , and deforming by isotopy, we obtain the upper half of figure 5. In the upper half of figure 5, the arc in the boundary of the shaded disk lying on the boundary of the right 3-ball is identified with the gray curve lying on the boundary of the left 3-ball. Then the branch set with respect to the quotient map $q$ appears as two strings in a 3-ball; see the lower half of figure 5 . That is, the pair $\left(M_{m, n} / \widehat{\iota}-q\left(N^{\circ}(\widehat{F})\right),\left(\left(M_{m, n}-\right.\right.\right.$ $\left.\left.\left.N^{\circ}(\widehat{F})\right) \cap \widehat{\alpha}\right) / \widehat{\iota}\right)$ gives a two-string tangle $T_{m, n}$ in $S^{3}$ such that the double branched covering space for this tangle is homeomorphic to $M_{m, n}-N^{\circ}(\widehat{F})$.

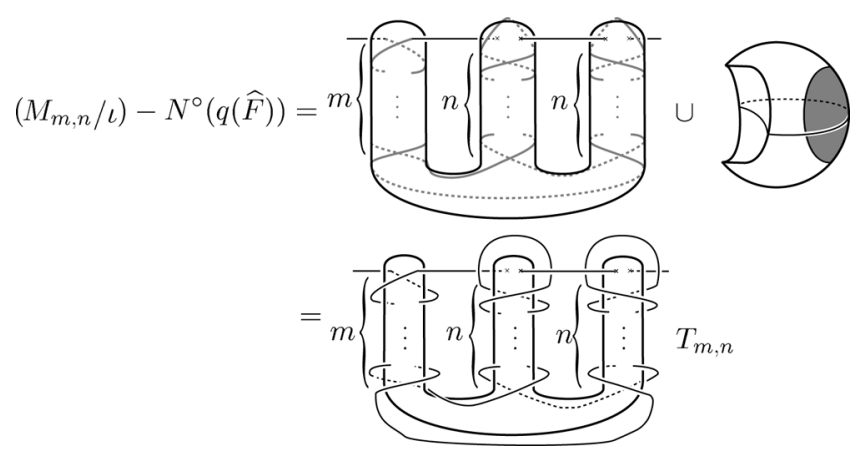

Figure 5: $\left(M_{m, n} / \widehat{\iota}-q\left(N^{\circ}(\widehat{F})\right),\left(\left(M_{m, n}-N^{\circ}(\widehat{F})\right) \cap \widehat{\alpha}\right) / \widehat{\iota}\right) \cong T_{m, n}$. 


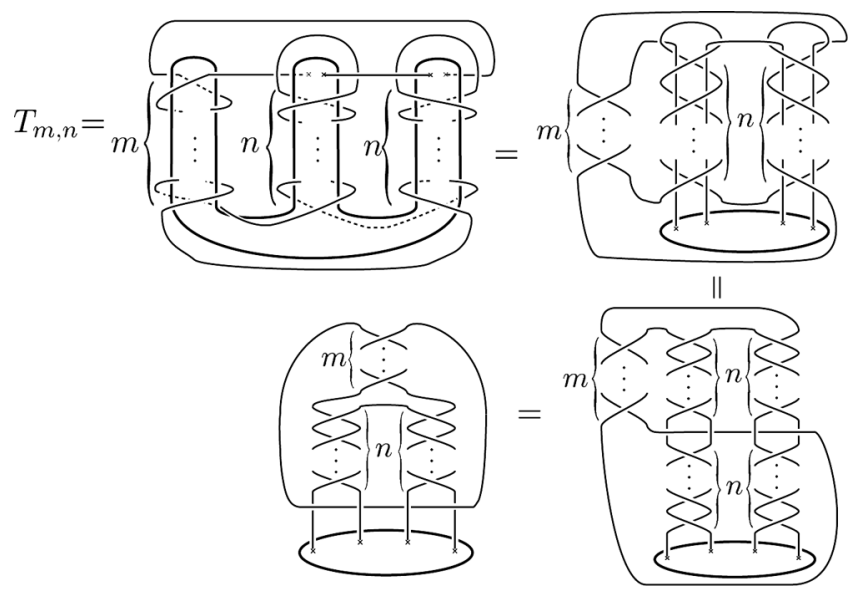

Figure 6: A deformation of $T_{m, n}$.

On the other hand, the tangle $T_{m, n}$ can be deformed as shown in figures 6 and 7 . Then taking the double-branched covering branched along $T_{m, n}$, we obtain the exterior of a knot, denoted by $J_{m, n}$, in $S^{2} \times S^{1}$; see figure 8 . From figure 8, we see that the knot $J_{m, n}$ is the closed braid of $\beta_{m, n}$, where $\beta_{m, n}=\sigma_{1} \cdots \sigma_{n} \sigma_{n+1}^{2 m-1} \sigma_{n+2}^{-1} \cdots \sigma_{2 n}^{-1} \in \mathbf{B}_{2 n+1}\left(S^{2}\right)$ (see figure 9). Now, we have the following.

Claim 3.3. The 3-manifold $K_{m, n}(0)-N^{\circ}(\widehat{F})$ is homeomorphic to the exterior $E\left(J_{m, n}\right)$ of $J_{m, n}$ in $S^{2} \times S^{1}$.

By Claims 3.1 to 3.3 , it suffices to show that the knot $J_{m, n} \subset S^{2} \times S^{1}$ has the exterior which is not Seifert fibered for $m \neq 0, n \geq 2$.

We start with the following claim.

Claim 3.4. The exterior $E\left(J_{ \pm 1, n}\right)$ of $J_{ \pm 1, n}$ in $S^{2} \times S^{1}$ is not Seifert fibered.

Proof. It suffices to show that $E\left(J_{1, n}\right)$ with $n \geq 2$ is not Seifert fibered since $E\left(J_{-1, n}\right)$ is orientation reversingly homeomorphic to $E\left(J_{1, n}\right)$. We show that if $E\left(J_{1, n}\right)$ is Seifert fibered, then $n=1$. Suppose that the exterior of $J_{1, n} \subset$ $S^{2} \times S^{1}$ admits a Seifert fibration. By [18, Lemma 4], this implies that $J_{1, n}$ is isotopic to a torus knot in $S^{2} \times S^{1}$. Note that the exponent sum of a $(2 n+1)$-string torus braid is congruent to 0 or $2 n$ modulo $4 n$. Thus, by Lemma 2.3, the exponent sum of $\beta_{1, n}$ must be congruent to 0 or $2 n$ modulo $4 n$. On the other hand, we see that $\beta_{1, n}$ has the exponent sum 2 and thus we have $n=1$. 

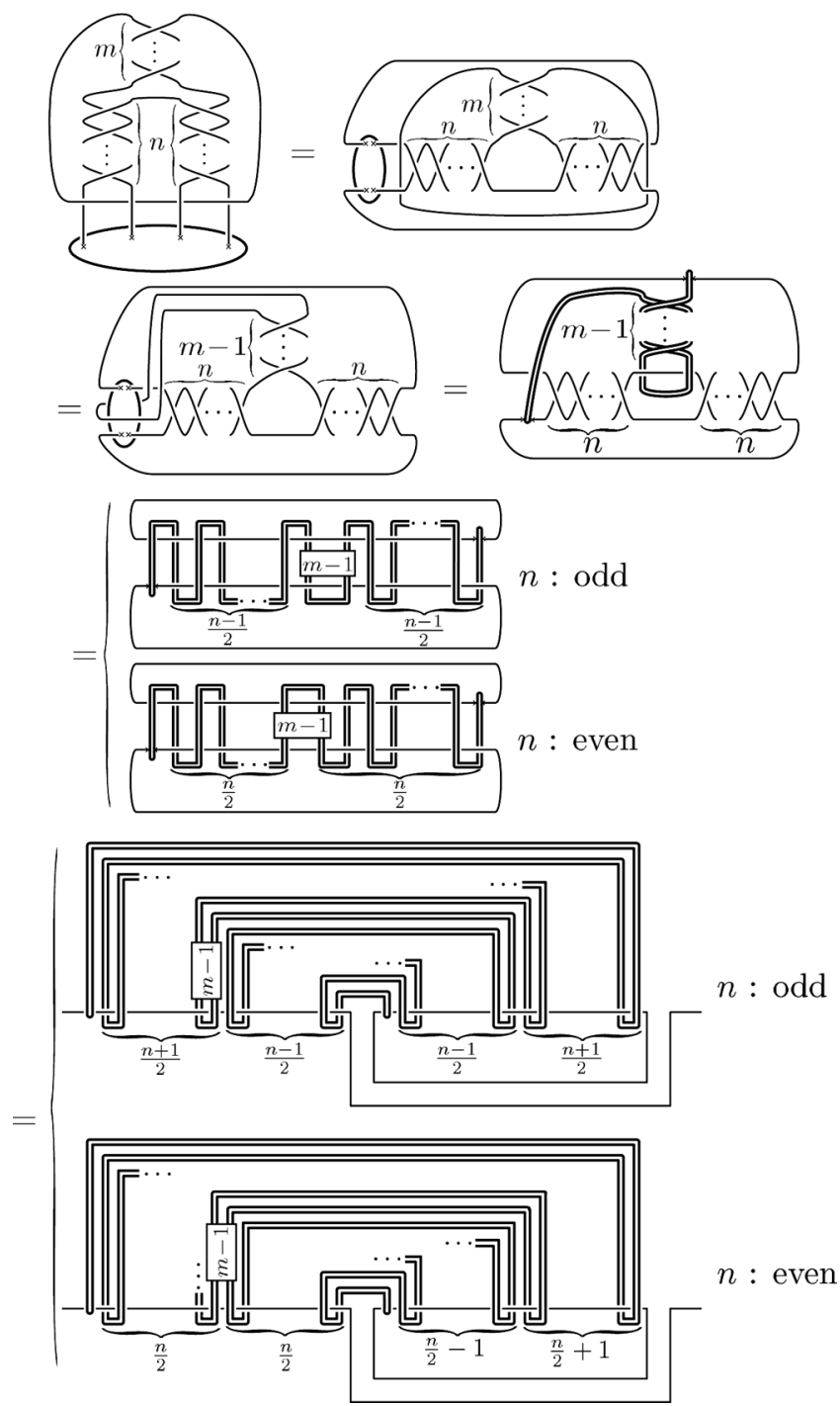

Figure 7: A deformation of $T_{m, n}$ (continued).

Here we note that the exterior of $J_{1, n}$ is atoroidal since the exterior of $K_{m, n}$ admits only one punctured torus up to isotopy [34]. Also note that the exterior of $J_{1, n}$ is irreducible since it is a $(2 n+1)$-punctured disk bundle over the circle. Therefore, Claim 3.4 implies that $J_{ \pm 1, n}$ is hyperbolic for $n \geq 2$.

We now consider the remaining case where $|m| \geq 2$ and $n \geq 2$ in the following two claims (Claims 3.5 and 3.6). In order to state these, let us 


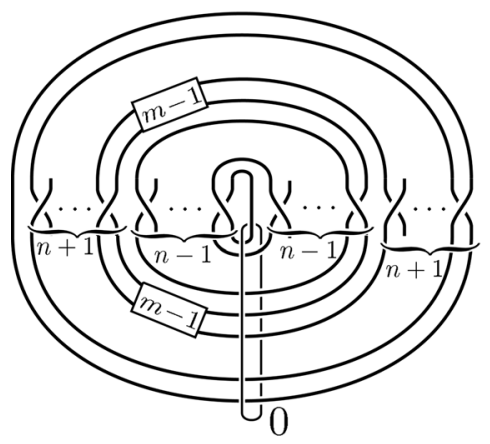

$n$ : odd

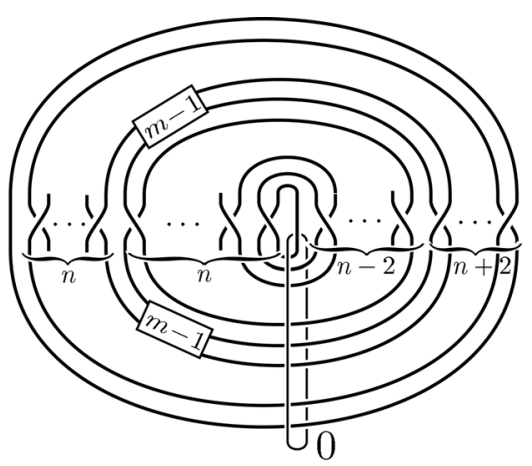

$n$ : even

Figure 8: $J_{m, n}=\widehat{\beta_{m, n}}$.

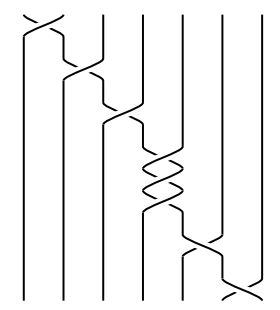

Figure 9: $\beta_{2,3}=\sigma_{1} \sigma_{2} \sigma_{3} \sigma_{4}^{3} \sigma_{5}^{-1} \sigma_{6}^{-1}$.

consider the augmented two-component link $L_{n}=J_{1, n} \cup U$ in $S^{2} \times S^{1}$ as shown in figure 10 . That is, $J_{1, n}$ is the knot considered in Claim 3.4, and $U$ is the trivial knot lying on a level sphere $S^{2} \times\{t\}$ such that the $-1 /(m-1)$ surgery on $U$ yields the knot $J_{m, n}$ (see figure 10).

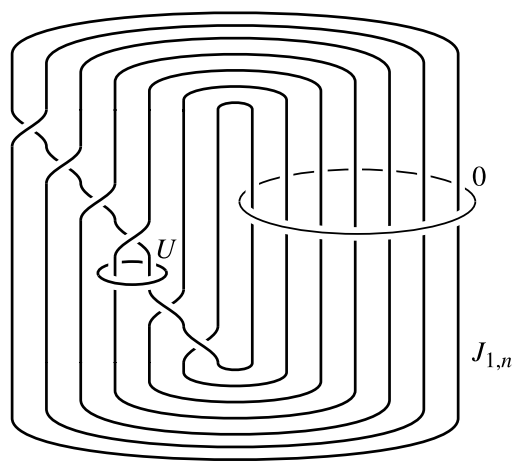

Figure 10: The case where $n=3 ; L_{3}$. 


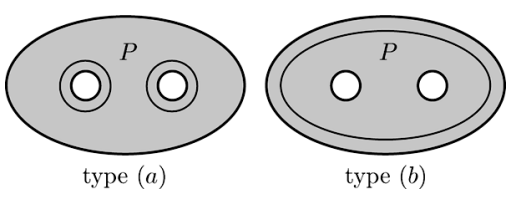

Figure 11: Essential loops on $P$.

Claim 3.5. If the link $L_{n}$ is hyperbolic, then $S^{2} \times S^{1}-N^{\circ}\left(J_{m, n}\right)$ is not Seifert fibered for integers $|m| \geq 2$ and $n \geq 2$.

Proof. Suppose that the link $L_{n}$ is hyperbolic. Assume for a contradiction that the exterior of $J_{m, n} \subset S^{2} \times S^{1}$ is Seifert fibered for some $|m| \geq 2$ and $n \geq 2$, and then $J_{-m, n}$ is also Seifert fibered. Notice that the knot $J_{m, n}$ is obtained from $J_{1, n}$ by twisting along $U$, equivalently, by the $-1 /(m-1)$ surgery on $U$. This means that both Dehn surgeries on $U$ along the slopes $-1 /(m-1)$ and $-1 /(-m-1)$ are Seifert fibered. By [11, Corollary 1.2], the distance of such a pair is at most three since the link $L_{n}$ is hyperbolic. This contradicts the condition on the distance of the slopes $-1 /(m-$ $1)$ and $-1 /(-m-1) ;|(-1)(-m-1)-(-1)(m-1)|=|m+1+m-1|=$ $2|m| \geq 4$.

Claim 3.6. The link $L_{n}$ is hyperbolic.

Proof. We show that $E\left(L_{n}\right)$ contains no essential sphere (i.e., $E\left(L_{n}\right)$ is irreducible), no essential disk (i.e., $E\left(L_{n}\right)$ is boundary irreducible), no essential torus (i.e., $E\left(L_{n}\right)$ is atoroidal) and $E\left(L_{n}\right)$ is not Seifert fibered in Subclaims 3.2 to 3.5 , respectively. Then, by [30], the link $L_{n}$ is hyperbolic.

First, we set the following notations used in the proof. Let $D_{U}$ be the disk bounded by $U$, which lies on a level sphere $S^{2} \times\{t\}$ and intersects $J_{1, n}$ transversely at just two points. Let $P$ be the twice-punctured disk appearing as the intersection $E\left(L_{n}\right) \cap D_{U}$, which is incompressible in $E\left(L_{n}\right)$ since it is an essential sub-surface of a fiber surface of $E\left(J_{1, n}\right)$. There are only three isotopy classes of essential loops on $P$, two of which are parallel to the meridian of $J_{1, n}$, and the other one is parallel to $U$. For simplicity, we say that the former are of type (a) and the latter is of type (b) (see figure 11).

The following subclaim is easily shown by figure 12 .

Subclaim 3.1. The exterior $E\left(L_{n} \cup D_{U}\right)$ of $L_{n} \cup D_{U}$ in $S^{2} \times S^{1}$ is homeomorphic to a handlebody of genus two. 


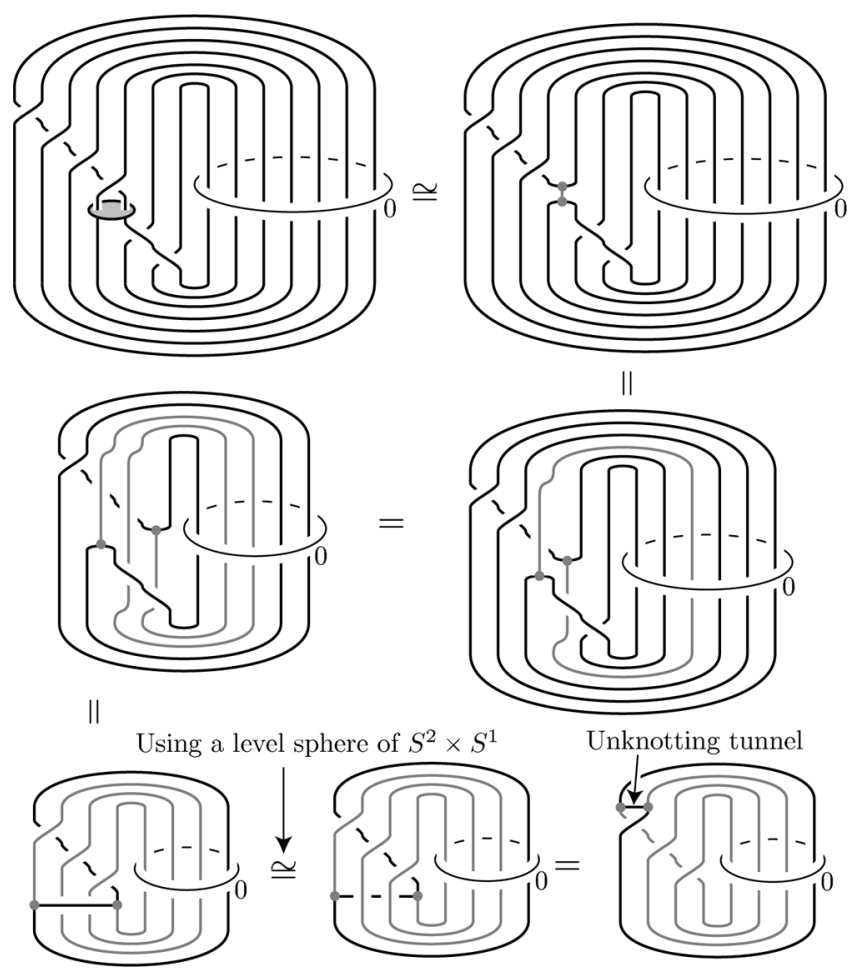

Figure 12: The case where $n=3$. Other cases are shown in a similar way.

We note that Subclaim 3.1 implies that $E\left(L_{n} \cup D_{U}\right)$ is irreducible and atoroidal.

Subclaim 3.2. The exterior $E\left(L_{n}\right)$ is irreducible.

Proof. Assume for a contradiction that $E\left(L_{n}\right)$ contains an essential sphere $S$, that is, $S$ does not bound a 3 -ball in $E\left(L_{n}\right)$. Then $S$ must have non-empty intersection with $P$ since, if otherwise, $E\left(L_{n} \cup D_{U}\right)$ contains an essential sphere, which contradicts Subclaim 3.1. Furthermore, $S$ can be isotoped so that the intersection $S \cap P$ consists of only essential loops on $P$ by Subclaim 3.1. Since each of the loops bounds a disk on $S$, it bounds a disk in $E\left(L_{n}\right)$. This contradicts that $P$ is incompressible in $E\left(L_{n}\right)$.

Subclaim 3.3. The exterior $E\left(L_{n}\right)$ is boundary irreducible.

Proof. Assume for a contradiction that $E\left(L_{n}\right)$ contains an essential disk $D$. Then, by compressing the torus component of $\partial E\left(L_{n}\right)$ along $D$, we have an 
embedded 2-sphere in $E\left(L_{n}\right)$. By Subclaim 3.2, the 2-sphere bounds a 3-ball, but this implies that $E\left(L_{n}\right)$ is homeomorphic to a 3-ball with a 1-handle, that is, a solid torus. A contradiction occurs.

Subclaim 3.4. The exterior $E\left(L_{n}\right)$ is atoroidal.

Proof. Assume for a contradiction that $E\left(L_{n}\right)$ contains an essential torus $T$. Then $T$ must have non-empty intersection with $P$ since, if otherwise, $E\left(L_{n} \cup\right.$ $\left.D_{U}\right)$ contains an essential torus, which contradicts Subclaim 3.1. We isotope $T$ so that the intersection $T \cap P$ consists of loops which are essential in both $T$ and $P$. This is possible since $T$ and $P$ are incompressible in $E\left(L_{n}\right)$ together with Subclaim 3.2. Then we have the following three cases:

(i) $T \cap P$ includes both of loops of type $(a)$ and $(b)$.

(ii) $T \cap P$ consists of loops of type (b) only.

(iii) $T \cap P$ consists of loops of type $(a)$ only.

Case (i): In this case, there exists a loop $c_{a}$ of type $(a)$ and a loop $c_{b}$ of type (b) in $T \cap P$, which cobounds an annulus $A_{a, b}$ on $T$, namely, $\partial A_{a, b}=c_{a} \cup c_{b}$. Then we have an embedded 2-sphere $S_{a, b}$ in $S^{2} \times S^{1}$, which is obtained from $A_{a, b}$ by capping of the boundaries by using the meridian disk bounded by $c_{a}$ and the sub-disk bounded by $c_{b}$ on $D_{U}$. Then $S_{a, b}$ intersects $J_{1, n}$ at just three points and thus $S_{a, b}$ is non-separating. This contradicts that $J_{1, n}$ is the closed $(2 n+1)$-braid on $S^{2}$ and $n \geq 2$.

Case (ii): Recall that $E\left(J_{1, n}\right)$ is atoroidal. Thus, $T$ must be either boundary parallel or compressible in $E\left(J_{1, n}\right)$.

Suppose first that $T$ is boundary parallel in $E\left(J_{1, n}\right)$. Then $T$ bounds a solid torus $V_{T}$ in $S^{2} \times S^{1}$ with $J_{1, n}$ as a core curve. Let $c_{b}$ be the component of $T \cap P$ which is innermost on $D_{U}$ among $T \cap P$. Then, since $T \cap P$ consists of loops of type $(b)$ only, $c_{b}$ bounds a disk in $D_{U}$ intersecting $J_{1, n}$ in two points. On the other hand, since $T$ bounds a solid torus $V_{T}$ in $S^{2} \times S^{1}$ with $J_{1, n}$ as a core curve, there is a compressing disk $D_{J}$ of $T$ which intersects $J_{1, n}$ exactly once. Note that $c_{b}$ and $\partial D_{J}$ are isotopic on $T$, and hence they must have the same linking number with $J_{1, n}$ in $V_{T}$, a contradiction.

Suppose next that $T$ is compressible in $E\left(J_{1, n}\right)$, namely, there exists a compressing disk $D_{T}$ for $T$ in $E\left(J_{1, n}\right)$. Note that $D_{T} \cap U \neq \emptyset$ since $T$ is incompressible in $E\left(L_{n}\right)$. Let $S_{T}$ be the 2-sphere embedded in $E\left(J_{1, n}\right)$ obtained from $T$ by compressing along $D_{T}$. Then, by irreducibility of $E\left(J_{1, n}\right)$, there exists a 3 -ball $B_{T}$ bounded by $S_{T}$ in $E\left(J_{1, n}\right)$. Let $Q_{T}$ be the compact sub-manifold of $E\left(J_{1, n}\right)$ with $\partial Q_{T}=T$. 
If $B_{T} \supset D_{T}$, then $B_{T}$ is obtained from $Q_{T}$ by attaching a 2-handle and thus $Q_{T}$ is obtained from $B_{T}$ by removing an open regular neighborhood of an arc properly embedded in $B_{T}$. Then $Q_{T}$ satisfies that $Q_{T} \cap D_{T}=$ $\partial Q_{T} \cap \partial D_{T}=T \cap \partial D_{T}$, and also satisfies that $Q_{T} \cap U=\emptyset$ since $D_{T} \cap U \neq$ $\emptyset$ and $T \cap U=\emptyset$. On the other hand, we find a sub-annulus $A_{U}$ on $D_{U}$ which connects $U$ and $T$ by the assumption that $T \cap P$ consists of loops of type (b) only. Let $N_{U}$ be a regular neighborhood of $Q_{T} \cup D_{T} \cup A_{U}$ in $E\left(J_{1, n}\right)$. Then $N_{U}$ is homeomorphic to a 3-ball such that $U \subset N_{U} \subset E\left(J_{1, n}\right)$. This means that there exists a 2-sphere $\partial N_{U}$ embedded in $E\left(L_{n}\right)$, separating the two boundary components. This contradicts Subclaim 3.2.

If $B_{T} \not \supset D_{T}$, then $Q_{T}$ is homeomorphic to a solid torus $V$ in $E\left(J_{1, n}\right)$, which is obtained from $B_{T}$ by attaching a 1-handle. Note that $D_{T}$ is a meridian disk in $V$. Also note that $U \subset V$ since $D_{T} \cap U \neq \emptyset$. Let $c$ be the loop in $T \cap P=T \cap D_{U}$ which is innermost on $D_{U}$. That is, $c$ bounds a disk $D$ on $D_{U}$ such that $D$ intersects $J_{1, n}$ at just two points and $D \cap T=\partial D \cap T=c$. Note that $c \subset T$ and $D \cap V=\partial D \cap \partial V=\partial D \cap T=c$. Then let us consider the intersection of $c$ and $\partial D_{T}$ on $T$.

If $c$ is isotopic to $\partial D_{T}$ on $\partial V=T$, then there exists the 2-sphere $S$ in $S^{2} \times S^{1}$ consisting of $D_{T}$ and $D$, which intersects $J_{1, n}$ at just two points. Notice that the algebraic intersection number between $S$ and $J_{1, n}$ is two and thus $S$ is non-separating. This contradicts that $J_{1, n}$ is the closed $(2 n+1)$ braid on $S^{2}$ and $n \geq 2$.

If the slope of $c$ and that of $\partial D_{T}$ have distance greater than one, i.e., the minimal geometric intersection number of their representatives is greater than one on $\partial V=T$, then a regular neighborhood of $V \cup D$ gives a punctured lens space $L(p, q)$ with $q>1$ embedded in $S^{2} \times S^{1}$, where $q$ is the distance between the slopes $c$ and $\partial D_{T}$. This contradicts that $S^{2} \times S^{1}$ is prime.

If the slopes of $c$ and $\partial D_{T}$ have distance just one on $\partial V=T$, then $U$ must be a core curve in $V$ as follows: let $c^{\prime} \subset T \cap P$ be the outermost loop on $D_{U}$. Since $c$ is parallel to $c^{\prime}$ on $T$ and $c^{\prime}$ is isotopic to $U$ in $V, c$ is isotopic to $U$ in $V$. This implies that $D_{T}$ is isotoped so that $U$ and $D_{T}$ intersects at a single point, meaning that $U$ is a core curve in $V$. Then $T=\partial V$ is boundary parallel in $E\left(L_{n}\right)$, contradicting that $T$ is essential in $E\left(L_{n}\right)$.

Case (iii): In this case, there must exist an incompressible annulus $A_{T}$ embedded in $E\left(L_{n}\right)$, which connects $T$ and $\partial N(U)$. Assume for a contradiction that there are no incompressible annulus embedded in $E\left(L_{n}\right)$ connecting $T$ and $\partial N(U)$. Then, by [31, Theorem 1], $T$ survives incompressible 
with finitely many exceptions after Dehn surgeries on $U$. Since $(-1 /(m-1))$-surgery on $U$ gives the exterior of the knot $J_{m, n}$, which is atoroidal for $m \neq 0$ and $n \geq 2$, the torus $T$ must be boundary parallel in $E\left(J_{m, n}\right)$ after all but finitely many Dehn surgeries on $U$. This implies that $T$ is separating in $E\left(J_{1, n}\right)$, and $U$ is contained in the component of the complement of $T$ containing $\partial E\left(J_{1, n}\right)$. Now cut $E\left(J_{1, n}\right)$ along $T$ to obtain a 3-manifold $N$ having two torus boundaries which correspond to $\partial N\left(J_{1, n}\right)$ and $T$. For brevity, we also denote the two boundaries of $N$ by the same symbol $\partial N\left(J_{1, n}\right)$ and $T$, respectively. These boundary tori are all incompressible in $N$, since they are all incompressible in $E\left(J_{1, n}\right)$. Now, by the assumption of the case, there is an annulus embedded in $N$ connecting $\partial N\left(J_{1, n}\right)$ and $T$. Then by [5, Theorem 2.4.3 (b)], after some Dehn filling on $\partial N\left(J_{1, n}\right)$, i.e., attaching a solid torus along $\partial N\left(J_{1, n}\right)$, the boundary $T$ must be incompressible in the resultant manifold, say $N^{\prime}$. On the other hand, by the assumption, $T$ must become parallel to $\partial N\left(J_{1, n}\right)$ after all but finitely many Dehn surgeries on $U$. Thus, $T$ must become compressible in the manifolds obtained from $N^{\prime}$ by all but finitely many Dehn surgeries on $U$. It then follows from [31, Theorem 1] again that there exists an incompressible annulus in $N \subset E\left(L_{n}\right)$ which connects $T$ and $\partial N(U)$. This contradicts the assumption.

Furthermore, since $T$ becomes inessential after any $(-1 /(m-1))$ surgery for $m \neq 0 \in \mathbb{Z}$, the boundary slope of $A_{T}$ on $\partial N(U)$ has distance one from any slope represented by $-1 /(m-1)$ [5, Theorem 2.4.3]. This means that the boundary slope of $A_{T}$ on $\partial N(U)$ must be $0 / 1$, namely, longitudinal.

Let $c_{A}$ be the loop $A_{T} \cap T$. If $c_{A}$ is trivial on $T$, then the disk bounded by $c_{A}$ with $A_{T}$ gives an essential disk in $E\left(L_{n}\right)$, contradicting to Subclaim 3.3. Hence $c_{A}$ is non-trivial on $T$. Now we further isotope $T$ so that the intersection $T \cap P$ has the minimal number of components.

Let us consider the intersection $c_{A} \cap P$. We may assume that $c_{A}$ and $P$ intersect transversely. Furthermore, we can isotope $A_{T}$ so that $c_{A} \cap P=\emptyset$ as follows. Assume that $c_{A} \cap P \neq \emptyset$. Since the boundary slope of $A_{T}$ on $\partial N(U)$ is longitudinal, $A_{T}$ is isotoped near $\partial N(U)$ so that $\left(A_{T} \cap \partial N(U)\right) \cap$ $(P \cap \partial N(U))=\emptyset$. Then there exists an arc $\alpha_{A} \subset A_{T} \cap P$ on $P$ such that $\partial \alpha_{A} \subset T \cap P$. Notice that $\alpha_{A}$ must be parallel to a sub-arc in $T \cap P$. For otherwise, $T$ is isotoped so that the number of components $T \cap P$ is reduced by using the bigon on $A_{T}$ bounded by $\alpha_{A}$ with a sub-arc on $\partial A_{T}$; see figure 13. This contradicts the assumption. Then, by the incompressibility of $T$ and Subclaim 3.2, we can isotope $A_{T}$ so that $\alpha_{A}$ vanishes. Repeating this process, $A_{T}$ is isotoped so that $c_{A} \cap P=\emptyset$. 


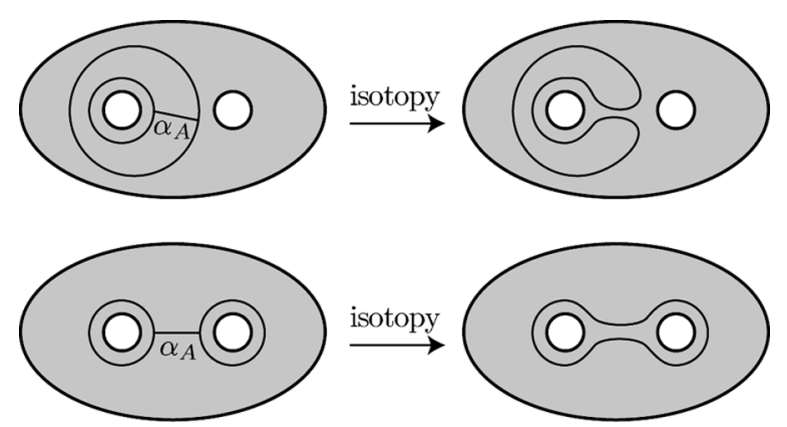

Figure 13: An isotopy of $T$ so that the number of components of $T \cap P$ is reduced.

Since $c_{A}$ is non-trivial and $c_{A} \cap P=\emptyset$, together with the assumption that $T \cap P$ consists of loops of type $(a)$ only, $c_{A}$ is parallel to the meridian of $J_{1, n}$.

Let us consider the intersection $A_{T} \cap P$. If $A_{T} \cap P=\emptyset$, then as in Case (i), we would find a 2 -sphere embedded in $S^{2} \times S^{1}$, which intersects $J_{1, n}$ at just three points. This contradicts that $J_{1, n}$ is the closed $(2 n+1)$-braid on $S^{2}$ and $n \geq 2$.

Now we consider the case where $A_{T} \cap P \neq \emptyset$. Then $A_{T} \cap P$ consists of loops on $P$ since all arc components vanish by the above argument. Since $A_{T}$ and $P$ are both incompressible in $E\left(L_{n}\right)$, together with Subclaim 3.2, the loops in $A_{T} \cap P$ are essential on both $A_{T}$ and $P$. This implies that all of the loops in $A_{T} \cap P$ are parallel on $A_{T}$. If the loop in $A_{T} \cap P$ nearest to $c_{A}$ on $A_{T}$ is of type $(b)$ on $P$, then, as in the case where $A_{T} \cap P=\emptyset$, we have a contradiction. If the loop in $A_{T} \cap P$ nearest to $\partial A_{T} \cap \partial N(U)$ is of type $(a)$ on $P$, then, we also have a contradiction in the same way. Otherwise, there must be a pair of loops in $A_{T} \cap P$ cobounding a subannulus on $A_{T}$, one of which is of type $(a)$ on $P$ and the other is of type $(b)$ on $P$. Again, in this case, we have a contradiction in the same way.

Now we complete the proof of Subclaim 3.4.

Subclaim 3.5. The exterior $E\left(L_{n}\right)$ is not Seifert fibered.

Proof. If $E\left(L_{n}\right)$ is Seifert fibered, then no Dehn surgery on $U$ gives a hyperbolic manifold. However, the trivial surgery on $U$ yields the exterior of $J_{1, n}$, which is hyperbolic.

By Subclaims 3.2 to 3.5, we complete the proof of Claim 3.6. 
Consequently, by Claims 3.1 to 3.6, we complete the proof of Lemma 2.1.

\section{On non-pretzel Montesinos knots}

In this section, we show the following:

Lemma 4.1. For the Montesinos knot $K_{ \pm}=M(-1 / 2,1 / 3,1 /(6 \pm 1 / 2))$, the surgered manifold $K_{ \pm}(0)$ is not Seifert fibered.

Proof. We divide our argument into two claims.

Claim 4.1. The surgered manifold $K_{+}(0)$ is not Seifert fibered.

Proof. As mentioned in Remark 2.1, the knot $K_{+}$is fibered. Let $F$ be a fiber surface for $E\left(K_{+}\right)$, and $h: F \rightarrow F$ a monodromy homeomorphism with respect to the fibration of $E\left(K_{+}\right)$, that is, $E\left(K_{+}\right) \cong F \times[0,1] /(x, 1) \sim$ $(h(x), 0)$. By the natural extension, the surgered manifold $K_{+}(0)$ is regarded as a surface bundle with the monodromy map $\hat{h}: \hat{F} \rightarrow \hat{F}$, where $\hat{F}$ denotes the closed surface obtained from $F$ by capping off. Consider the induced isomorphism $\hat{h}_{*}: H_{1}(\hat{F}) \rightarrow H_{1}(\hat{F})$ from the monodromy $\hat{h}$. Notice that the characteristic polynomial of $\hat{h}_{*}$ coincides with the Alexander polynomial of $K_{+}$(see, for example,[28, p. 326, Corollary 8]). On the other hand, if the characteristic polynomial of $\hat{h}_{*}$ has no roots of unity as zeros, then the homeomorphism $h$ is not periodic [4, Lemma 5.1]. These imply that if $\Delta_{K_{+}}(t)$ has no root of unity as zeros, then $K_{+}(0)$ is not Seifert fibered. Actually, we have $\Delta_{K_{+}}(t)=-\left(t^{2}+t^{-2}\right)+3$, and then $\Delta_{K_{+}}(t)$ has no roots of unity as zeros. This completes the proof of Claim 4.1 .

In the remaining part, we will consider Dehn surgeries on links in $S^{3}$, equivalently, surgery descriptions for 3-manifolds. We omit the details. See [28, Chapter 9] for example.

Claim 4.2. The surgered manifold $K_{-}(0)$ is not Seifert fibered.

Proof. As illustrated in figure 14, the knot $K_{-}$with the surgery coefficient 0 is transformed into the link $L$ with the surgery coefficients $\mathbf{r}=$ $(0,1,1,-1,-1,-1 / 3)$ by the Kirby moves.

Then, as seen in [12, section 3], the 3 -manifold $L(\mathbf{r})$ obtained by the Dehn surgery on $L$ with surgery coefficients $\mathbf{r}$ is homeomorphic to a surface 

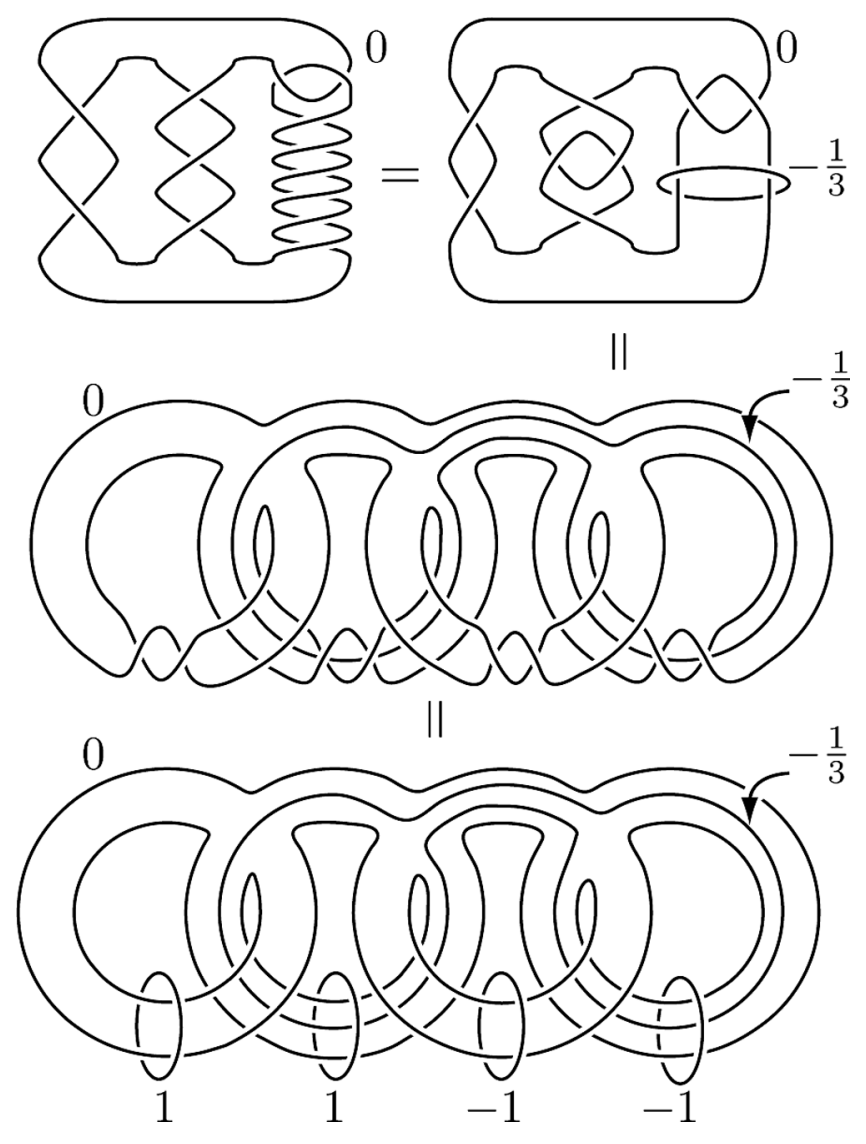

Figure 14: The Kirby moves from $\left(K_{-}, 0\right)$ to $(L, \mathbf{r})$.

bundle $M_{\varphi}$ over the circle with genus two surface fibers, and its monodromy $\operatorname{map} \varphi$ is described as

$$
\varphi=\tau_{5}^{3} \circ \tau_{3} \circ \tau_{1}^{-1} \circ \tau_{4} \circ \tau_{2}^{-1}
$$

Here $\tau_{i}(1 \leq i \leq 5)$ denotes the Dehn twist on the genus two surface along the simple closed curve $c_{i}$ depicted in figure 15 .

Furthermore, since $\varphi$ commutes with the hyper-elliptic involution, the manifold $L(\mathbf{r})$ is also obtained as the double-branched covering space of $S^{2} \times S^{1}$ branched along the 6 -string closed braid $\hat{\beta}$ in $S^{2} \times S^{1}$ with

$$
\beta=\sigma_{5}^{3} \sigma_{3} \sigma_{1}^{-1} \sigma_{4} \sigma_{2}^{-1} \in \mathbf{B}_{6}\left(S^{2}\right)
$$




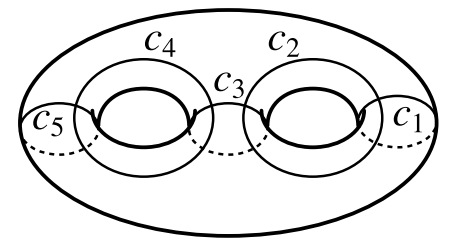

Figure 15: The simple closed curves $c_{1}, c_{2}, c_{3}, c_{4}, c_{5}$ on a genus two surface.

Now, assume for a contradiction that $K_{-}(0) \cong M_{\varphi}$ is Seifert fibered. Then, since the fiber surface is of genus two, by [16, VI. 31. Lemma], the monodromy map $\varphi$ must be periodic. This implies that the braid $\beta$ is of finite order in $\mathbf{B}_{6}\left(S^{2}\right)$. Then, by [22, Theorem 4.2], $\beta$ is conjugate to $\Delta^{k}$ for some integer $k$, where $\Delta$ denotes $\sigma_{1} \sigma_{2} \sigma_{3} \sigma_{4} \sigma_{5}$. This implies that the closed braid for $\beta$ is isotopic to that for $\Delta^{k}$. However, the exponent sum of $\beta$ is 3 , and that of $\Delta^{m}$ is $5 \mathrm{~m}$. Since $3 \not \equiv 5 m \bmod 10$, this contradicts Lemma 2.3. This completes the proof of Claim 4.2.

By Claims 4.1 and 4.2, we complete the proof of Lemma 2.2.

Remark 4.1. Unfortunately, the argument in the proof of Claim 4.1 (resp. Claim 4.2) does not work for proving Claim 4.2 (resp. Claim 4.1). Thus, we divide the proof into two cases according to their signs, and apply different methods.

\section{Acknowledgments}

K.I. and I.D.J. would like to thank Professor Makoto Sakuma for letting them know the result obtained by Professor Kunio Murasugi in [22]. They also thank the referees for their suggestions and comments, in particular, on improving the proofs of Claims 3.1 and Subclaim 3.4. K.I. is partially supported by Grant-in-Aid for Young Scientists (B), No. 20740039, Ministry of Education, Culture, Sports, Science and Technology, Japan. I.D.J. is partially supported by Grant-in-Aid for Research Activity Start-up, No. 22840037, Japan Society for the Promotion of Science.

\section{References}

[1] F. Bonahon and L.C. Siebenmann, Geometric splittings of knots, and Conway's algebraic knots, Draft of a monograph, 1979-1985. 
[2] F. Bonahon and L.C. Siebenmann, New geometric splittings of classical knots and the classification and symmetries of arborescent knots, preprint (2010).

[3] S. Boyer, Dehn surgery on knots, Handbook of Geometric topology, North-Holland, Amsterdam, 2002, ch. 4, 165-218

[4] A.J. Casson and S.A. Bleiler, Automorphisms of surfaces after Nielsen and Thurston, London Mathematical Society Student Texts, vol. 9, Cambridge University Press, Cambridge, 1988.

[5] M. Culler, C. McA. Gordon, J. Luecke and P.B. Shalen, Dehn surgery on knots, Ann. Math. 125(2) (1987), no. 2, 237-300.

[6] M. Eudave-Muñoz, On hyperbolic knots with Seifert fibered Dehn surgeries, Topology Appl. 121 (2002), no. 1-2, 119-141.

[7] D. Gabai, Detecting fibred links in $S^{3}$, Comment. Math. Helv. 61(4) (1986), 519-555.

[8] F. González-Acuña, Dehn's construction on knots, Bol. Soc. Mat Mexicana 15(2) (1970), 58-79.

[9] F. González-Acuña and H. Short, Knot surgery and primeness, Math. Proc. Cambridge Philos. Soc. 99(1) (1986), 89-102.

[10] C.McA. Gordon and J. Luecke, Seifert fibered surgeries on hyperbolic knots, Abstracts Am. Math. Soc. 20 (1999).

[11] C.McA. Gordon and Y.Q. Wu, Annular Dehn fillings, Comment. Math. Helv. 75 (2000), 430-456.

[12] K. Ichihara, On framed link presentations of surface bundles, J. Knot Theory Ramifications 7(8) (1998), 1087-1092.

[13] K. Ichihara and I.D. Jong, Cyclic and finite surgeries on Montesinos knots, Algebr. Geom. Topol. 9(2) (2009), 731-742.

[14] K. Ichihara, I.D. Jong and S. Mizushima, Seifert fibered surgeries on alternating Montesinos knots, preprint (2010).

[15] K. Ichihara, K. Motegi, and H.-J. Song, Seifert fibered slopes and boundary slopes on small hyperbolic knots, Bull. Nara Univ. Ed. Natur. Sci. $\mathbf{5 7}(2)$ (2008), 21-25.

[16] W. Jaco, Lectures on three-manifold topology, CBMS Regional Conference Series in Mathematics, 43, American Mathematical Society, Providence, RI, 1980. 
[17] A. Kawauchi, A Survey of knot theory, Birckhäuser-Verlag, Basel, 1996.

[18] P. Kohn, Two-bridge links with unlinking number one, Proc. Am. Math. Soc. 113(4) (1991), 1135-1147.

[19] J.M. Montesinos, Surgery on links and double branched covers of $S^{3}$, Knots, groups, and 3-manifolds (Papers dedicated to the memory of R. H. Fox) Ann. Math. Studies, 84 (1975), 227-259.

[20] L. Moser, Elementary surgery along a torus knot, Pacific J. Math. 38 (1971), 737-745.

[21] K. Motegi, Dehn surgeries, group actions and Seifert fiber spaces, Comm. Anal. Geom. 11(2) (2003), 343-389.

[22] K. Murasugi, Seifert fibre spaces and braid groups, Proc. London Math. Soc. (3) 44(1) (1982), 71-84.

[23] K. Murasugi and B.I. Kurpita, A study of braids, Kluwer Academic Publishers, Dordrecht, 1999.

[24] U. Oertel, Closed incompressible surfaces in complements of star links, Pacific J. Math. 111(1) (1984), 209-230.

[25] G. Perelman, The entropy formula for the Ricci flow and its geometric applications, arXiv:math.DG/0211159 (2002).

[26] G. Perelman, Ricci flow with surgery on three-manifolds, arXiv:math.DG/0303109 (2003).

[27] G. Perelman, Finite extinction time for the solutions to the Ricci flow on certain three-manifolds, arXiv:math.DG/0307245 (2003).

[28] D. Rolfsen, Knots and links, Mathematics Lecture Series, no. 7, Publish or Perish Inc., Berkeley, California, 1976.

[29] W.P. Thurston, The geometry and topology of 3-manifolds, Lecture notes, Princeton University, 1978, electronic version available at http://www.msri.org/publications/books/gt3m.

[30] W.P. Thurston, Three-dimensional manifolds, Kleinian groups and hyperbolic geometry, Bull. Am. Math. Soc. 6(3) (1982), 357-381.

[31] Y.-Q. Wu, Incompressibility of surfaces in surgered 3-manifolds, Topology 31(2) (1992), 271-279.

[32] Y.-Q. Wu, Dehn surgery on arborescent knots, J. Differential Geom. 43(1) (1996), 171-197. 
[33] Y.-Q. Wu, Dehn surgery on arborescent knots and links - a survey, Chaos Solitons Fractals 9(4-5) (1998), 671-679.

[34] Y.-Q. Wu, The classification of toroidal Dehn surgeries on Montesinos knots, preprint, math.GT/0610870 (2006).

Department of Mathematics

College of Humanities and Sciences, Nihon University

3-25-40 Sakurajosui, Setagaya-Ku, Tokyo 156-8550, Japan

E-mail address: ichihara@math.chs.nihon-u.ac.jp

Osaka City University Advanced Mathematical Institute

3-3-138, Sugimoto, Sumiyoshi-ku, Osaka, 558-8585, Japan

E-mail address: jong@sci.osaka-cu.ac.jp

Received May 13, 2010

This paper is dedicated to Professor Kunio Murasugi on the occasion of his 80th birthday. 